\title{
Activation of an Endogenous Suicide Response after Perturbation of rRNA Synthesis Leads to Neurodegeneration in Mice
}

\author{
Rosanna Parlato, ${ }^{1}$ Grzegorz Kreiner, ${ }^{1}$ Gitta Erdmann, ${ }^{1}$ Claus Rieker, ${ }^{1}$ Stefanie Stotz, ${ }^{1}$ Ella Savenkova, ${ }^{1}$ Stefan Berger, ${ }^{1}$ \\ Ingrid Grummt, ${ }^{2}$ and Günther Schütz ${ }^{1}$ \\ ${ }^{1}$ Division of Molecular Biology of the Cell I and 2Division of Molecular Biology of the Cell II, German Cancer Research Center, Deutsches \\ Krebsforschungszentrum-Zentrum für Molekulare Biologie der Universität Heidelberg Alliance, D-69120 Heidelberg, Germany
}

\begin{abstract}
Transcription of rRNA genes is essential for maintaining nucleolar integrity, a hallmark for the healthy state and proliferation rate of a cell. Inhibition of rRNA synthesis leads to disintegration of the nucleolus, elevated levels of $\mathrm{p} 53$, and induction of cell suicide, identifying the nucleolus as a critical stress sensor. Whether deregulation of rRNA synthesis is causally involved in neurodegeneration by promoting cell death and/or by inhibiting cellular growth has however not been addressed. The transcription factor TIF-IA plays a central role in mammalian rRNA synthesis, regulating the transcriptional activity of RNA polymerase I. To investigate the consequences of nucleolar perturbation in the nervous system, we have chosen to specifically ablate the gene encoding the transcription factor TIF-IA in two different contexts: neural progenitors and hippocampal neurons. Here, we show that ablation of TIF-IA leads to impaired nucleolar activity and results in increased levels of the proapoptotic transcription factor p53 in both neural progenitors and hippocampal neurons but induces rapid apoptosis only in neural progenitors. Nondividing cells of the adult hippocampus are more refractory to loss of rRNA transcription and face a protracted degeneration. Our study provides an unexploited strategy to initiate neurodegeneration based on perturbation of nucleolar function and underscores a novel perspective to study the cellular and molecular changes involved in the neurodegenerative processes.
\end{abstract}

Key words: survival; rRNA transcription; neural progenitors; hippocampus; degeneration; nucleolus

\section{Introduction}

As the regulation of ribosome number is critical for cell growth and proliferation, rRNA genes (rDNA) are transcribed with high efficiency to keep up with the cell's metabolic activity and demand of ribosomes. Given the enormous energetic demand of this process, rDNA transcription by RNA polymerase I (Pol I) is tightly regulated in response to changes in growth and specific environmental challenges, such as growth factors, drugs, stress, or nutrient availability (Grummt, 2003). The key factor that transduces extracellular signals to the Pol I transcription apparatus is the transcription initiation factor TIF-IA, a Pol I-specific transcription initiation factor that plays a central role in the regulation of rDNA transcription. External signals that affect cell growth and proliferation alter the phosphorylation pattern of

\footnotetext{
Received May 30, 2008; revised Aug. 19, 2008; accepted 0ct. 8, 2008.

This work was supported by the Deutsche Forschungsgemeinschaft through Collaborative Research Centres Sonderforschungsbereich (SFB) 488 and SFB 636, Forschergruppe 0t 165/2-2, Graduiertenkolleg 791/1.02, and Sachbeihilfe Schu 51/7-2; the Fonds der Chemischen Industrie; the European Union through Grant LSHM-CT-2005018652 (CRESCENDO); the Bundesministerium für Bildung und Forschung through Nationales Genomforschungsnetz Grants FZK 01GS01117, 01GS0477, and KGCV1/01GS0416; German-Polish Cooperation project 01GZ0310; and Systems Biology projects number 0313074 C (HepatoSys) and NW5 (CoReNe). We thank the Carl Zeiss Application Center in the Heidelberg Technology Park for confocal laser-scanning microscopy.

Correspondence should be addressed to Dr. Günther Schütz, Division of Molecular Biology of the Cell I, German Cancer Research Center, D-69120 Heidelberg, Germany. E-mail: g.schuetz@dkfz.de.

DOI:10.1523/JNEUROSCI.2439-08.2008

Copyright $\odot 2008$ Society for Neuroscience $\quad$ 0270-6474/08/2812759-06\$15.00/0
}

TIF-IA and modulate TIF-IA activity to up or downregulate cellular pre-rRNA synthesis, ribosome biogenesis and cell proliferation (Mayer et al., 2005). Consistent with TIF-IA playing a key role in the regulatory networks that control cell growth and proliferation, a germline mutation of the TIF-IA gene results in massive cell death leading to early embryonic lethality (Yuan et al., 2005). Experiments in murine embryonic fibroblasts (MEFs) have shown that loss of TIF-IA blocks cell cycle progression and augments cell death. Importantly, cell cycle arrest and apoptosis of TIF-IA-deficient cells was dependent on elevated levels of the transcription factor p53, supporting the view that the nucleolus is a stress sensor that regulates the abundance of p53 (Rubbi and Milner, 2003; Olson, 2004).

While the central role of TIF-IA in growth-dependent regulation of Pol I activity is very well characterized, the relevance of deregulation of rRNA synthesis in the vertebrate nervous system is still unknown. Interestingly, a recent study in cultured cortical neurons showed that nucleolar perturbation induced by DNA damage triggers neuronal apoptosis (Kalita et al., 2008). Here, we have asked whether loss of TIF-IA and thereby deregulation of rRNA synthesis plays a role in the developing and adult nervous system. We took advantage of specific Cre recombinase transgenic mouse lines allowing the conditional inactivation of the TIF-IA gene either in embryonic neural progenitors or in adult hippocampal neurons using the Cre/loxP system in mice 
(Tronche et al., 1999; Erdmann et al., 2007). Our study shows a differential impact of TIF-IA inactivation on the survival of proliferating and postmitotic neurons in vivo. While neural progenitors rapidly die, postmitotic neurons survive for longer periods despite inhibition of rRNA synthesis. Our study provides the first in vivo evidence that perturbation of nucleolar activity initiates neurodegeneration by activation of an endogenous response leading to p53 activation.

\section{Materials and Methods}

Generation of transgenic mice and treatment with tamoxifen. Mice carrying the TIF-IA floxed allele (Yuan et al., 2005) were crossed to the transgenic mouse lines NestinCre (Tronche et al., 1999) or CaMKCreER ${ }^{T 2}$ (Erdmann et al., 2007) to generate TIF-IA ${ }^{+/ f l}$ Cre positive mice. Mutant mice were generated by crossing TIF-IA ${ }^{+/ f l}$; Cre mice with TIF-IA ${ }^{\text {fl/fl }}$ mice. The analysis of the genotype was performed as previously described (Tronche et al., 1999; Yuan et al., 2005; Erdmann et al., 2007). Mice were intraperitoneally injected with tamoxifen (Sigma) at the age of 3 months as previously described (Erdmann et al., 2007).

Immunohistochemistry and in situ hybridization. Embryos and brains were fixed in $4 \%$ paraformaldehyde, $\mathrm{pH} 7.2$, overnight and processed for either paraffin or vibratome sections. Paraffin sections $(7 \mu \mathrm{m})$ were treated for Nissl staining with cresyl violet. For immunohistochemistry, the following primary antibodies were used: anti-NPM/B23 (1:2000, Millipore), anti-p53 (1:800, Novocastra), anti-cleaved caspase-3 (Asp175) antibody (1:800, Cell Signaling Technology), anti-NeuN (1: 3000 , Millipore). The sections were incubated in citrate buffer, $\mathrm{pH} 6.0$, and boiled in a microwave oven. The primary antibodies were incubated overnight at $4^{\circ} \mathrm{C}$. Biotin-conjugated secondary antibody was diluted 1:400 in PBS and detection was performed using the avidin-biotin system (Vector Laboratories) with the VECTOR peroxidase kit. The staining was developed with $\mathrm{DAB}$ and $\mathrm{H}_{2} \mathrm{O}_{2}$ (Sigma-Aldrich). For double immunofluorescence, sections were incubated with anti-NPM/B23 (1:500) at $4^{\circ} \mathrm{C}$ overnight, followed by goat anti-mouse IgG coupled with Alexa Fluor 594 (Invitrogen) for $1 \mathrm{~h}$ at room temperature and incubated again with anti-p53 (1:100) at $4^{\circ} \mathrm{C}$ overnight, followed by goat anti-rabbit IgG coupled with Alexa Fluor 488 (Invitrogen) for $1 \mathrm{~h}$ at room temperature. Fluorescent granule cells in the dentate gyrus (DG) of the hippocampus and pyramidal cells of CA1 and CA3 were viewed under a confocal laserscanning microscope (LSM710, Carl Zeiss MicroImaging).

Nonradioactive in situ hybridization was performed on paraffin sections as previously described (Parlato et al., 2004). The presence of 47Sand $28 \mathrm{~S}$ rRNA was analyzed by using three specific riboprobes contained in the leader and the $28 \mathrm{~S}$ sequences as described (Qian et al., 2006).

Terminal deoxynucleotidyl transferase-mediated biotinylated UTP nick end labeling-(TUNEL) staining was performed according to the manufacturer's instructions (in situ cell death detection kit, POD, Roche).

Fluoro-Jade B staining (Schmued and Hopkins, 2000) was performed on coronal vibratome sections (50 $\mu \mathrm{m})$ according to the manufacturer's recommendation (Millipore).

Cell count and statistics. Neuronal density in all three hippocampal subfields was measured on light-microscopy images after immunostaining coronal paraffin sections $(7 \mu \mathrm{m})$ by counting the number of NeuN positive cells $/ \mathrm{mm}^{2}$ in five separated areas per each subfield (DG, CA1 and CA3). The quantification of TUNEL-positive cells was done from every sixth, $7 \mu \mathrm{m}$ section through the hippocampus. Positive cells were counted in one-half (hemisection) of the hippocampus. Reported is the average value of 3-4 mice per genotype. Bar represents \pm SEM. Statistical significance was analyzed using a homoscedastic Student's $t$ test. Values were considered significantly different with ${ }^{\star} p<0.05,{ }^{* *} p<0.01,{ }^{* * *} p<$ 0.001 .

\section{Results}

\section{TIF-IA is essential for survival of embryonic neural progenitors}

To dissect the function of TIF-IA in the developing nervous system, mice carrying the floxed allele of the TIF-IA gene (Yuan et al., 2005) were crossed with the NestinCre transgenic mice, expressing the Cre recombinase in neural and glial progenitors as early as E9.5 (Tronche et al., 1999). The TIF-IA ${ }^{f l f l}$; NestinCre (abbreviated TIF-IA ${ }^{\text {NesCre }}$ ) mutants are perinatally lethal. Despite the fact that their body size is comparable with control littermates, the TIF-IA ${ }^{\text {NesCre }}$ mutants lack virtually the entire brain. Moreover the head shows an excess of CSF (hydroanencephaly) and residual membranes compared with control. To characterize the events compromising so severely brain development, we have analyzed control and mutant mice at different embryonic stages (E9.5, E11.5 and E14.5). At E9.5 the morphology of the neural tube is similar in controls and TIF-IA $A^{\text {NesCre }}$ mutants (data not shown). Sections through the developing brain revealed the first visible changes at E11.5, mainly in the ganglionic eminences (GE) and in the hindbrain where many pyknotic nuclei are readily detectable in the ventricular zone of mutants (Fig. 1A,B). At E14.5 the overall organization of the embryonic brain is severely damaged (Fig. 1C,D). Histological analysis of the head of newborn mutants by Nissl staining showed that no brain tissue is left (Fig. 1E,F).

To study the cellular and molecular changes occurring in TIF$I A^{\text {NesCre }}$ mutants, we focused our analysis on the ganglionic eminences at E11.5. As previous studies have established that the nucleolar structure is perturbed in TIF-IA-deficient mice (Yuan et al., 2005), we monitored nucleolar integrity in brain of TIF$I A^{\text {NesCre }}$ mice using an antibody that recognizes nucleophosmin (NPM/B23), a nucleolar phosphoprotein required for assembly of ribosomes. In control mice, NPM/B23-positive nucleoli are visible as distinct punctuate structures within the GEs in the region underlying the ventricle (Fig. $1 G, G^{\prime}$ ). In mutant mice, however, NPM/B23 is distributed throughout the nucleoplasm (Fig. $\left.1 \mathrm{H}, \mathrm{H}^{\prime}\right)$, demonstrating that the nucleolar structure is perturbed (Borer et al., 1989; Kalita et al., 2008). Disintegration of nucleolar structure in the GEs of TIF-IA NesCre mutants was accompanied by elevated levels of p53 immunoreactivity (Fig. $1 I, K$ ). This increase in p53 levels is associated with a marked increase in apoptotic cells in this area, as detected by TUNEL assay (data not shown) and enhanced caspase-3 activity, a marker of apoptosis (Fig. $1 \mathrm{~L}$ ). Neither staining was observed in the same regions of control littermates (Fig. $1 \mathrm{~J}$, not shown).

\section{Perturbation of pre-rRNA synthesis in the adult leads to degeneration of hippocampal neurons}

To investigate the effects of TIF-IA ablation in the adult brain, we have used a transgenic mouse line expressing the Cre recombinase only in neurons under control of the calcium/calmodulindependent protein kinase II $\alpha$ (CaMKII $\alpha$ ) gene regulatory elements (Erdmann et al., 2007). To achieve specific recombination of the TIF-IA alleles in adulthood, the Cre recombinase was fused with an altered ligand binding domain of the estradiol receptor $\left(\mathrm{ER}^{\mathrm{T} 2}\right)$, so that only upon tamoxifen treatment, the fusion protein $\left(\mathrm{CreER}^{\mathrm{T} 2}\right)$ translocates into the nucleus and triggers DNA recombination. Mice carrying the floxed TIF-IA allele TIF-IA fl/fl (Yuan et al., 2005) were crossed with CaMKCreER ${ }^{T 2}$ transgenic mice to yield TIF-IA CaMKCreERT2 mutant mice. The activity of the transgene can be successfully induced by intraperitoneal administration of tamoxifen in the adult forebrain: cortex, amygdala, hippocampus (Erdmann et al., 2007). To monitor the effect of TIF-IA inactivation on rDNA transcription, we have analyzed control and mutant mice at 2 weeks, 4 weeks, and 3 months after injection of tamoxifen in the hippocampus (Fig. $2 \mathrm{~A}$ ). Transcriptional activity was monitored by in situ hybridization with a riboprobe specific for the $47 \mathrm{~S}$ rRNA precursor and located in the 

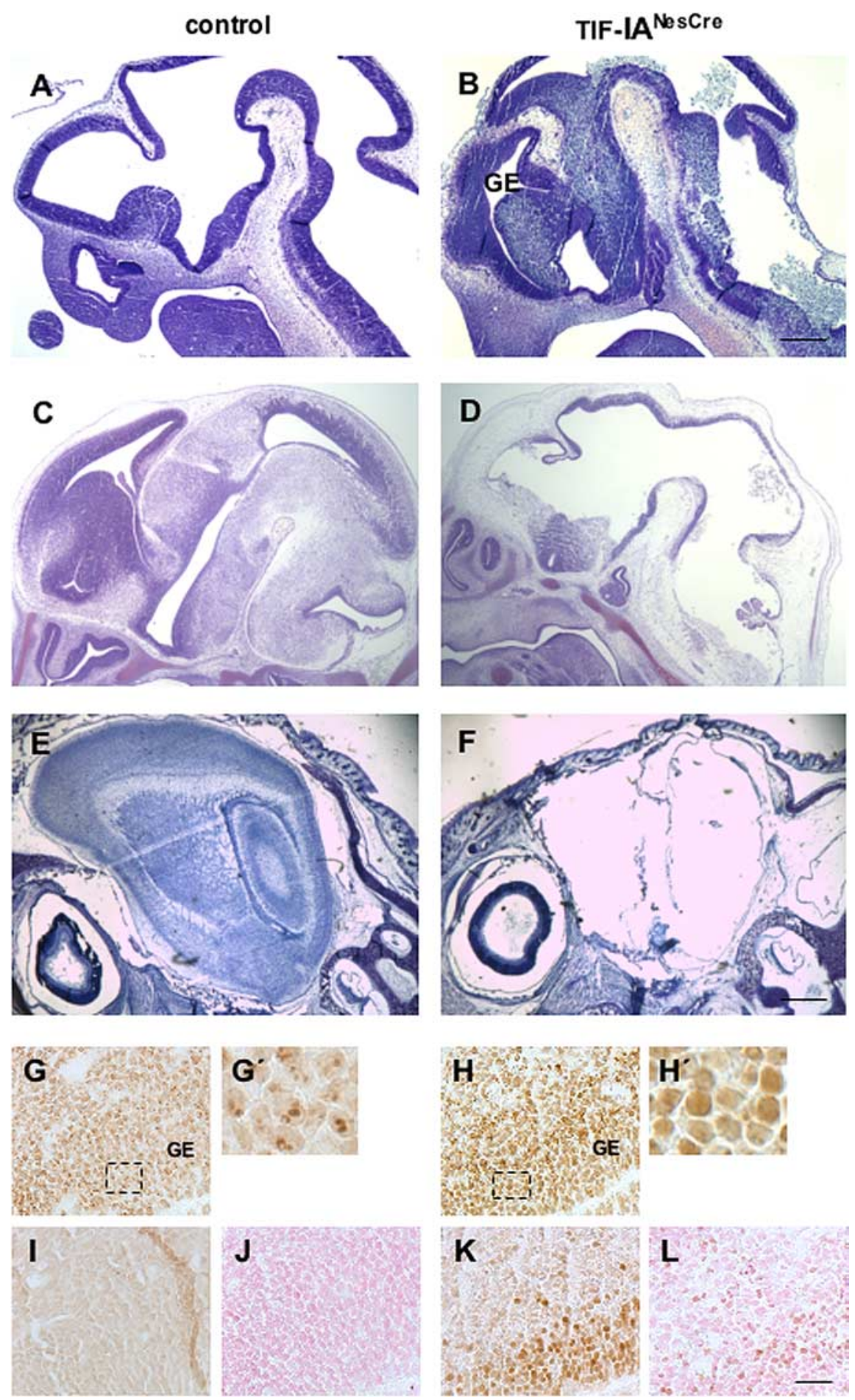

Figure 1. Neural progenitors lacking TIF-IA undergo apoptotic cell death. $\boldsymbol{A}-\boldsymbol{F}$, Sagittal paraffin sections from control $(\boldsymbol{A}, \boldsymbol{C}, \boldsymbol{E})$ and $T I F-I A^{\text {Nescre }}$ mutant $(\boldsymbol{B}, \boldsymbol{D}, \boldsymbol{F})$ analyzed by Nissl staining at different developmental stages (E11.5, E14.5 and newborn) show the anatomical changes leading to mice born without a brain. $\boldsymbol{G}, \boldsymbol{H}$, Immunohistochemistry with NPM/B23 specific antibody is used to analyze the nucleoli in control $\left(\mathbf{G}, \boldsymbol{G}^{\prime}\right)$ and in the TIF-I $A^{\text {Nes } r \text { re }}$ mutant $\left(\boldsymbol{H}, \boldsymbol{H}^{\prime}\right)$ at E11.5. $\boldsymbol{I}-\boldsymbol{L}, 0$ verexpression of p53 is observed within the GE of the TIF-IA Nes(re mutant $(\boldsymbol{K})$ but not in the control $(\boldsymbol{I})$. Immunostaining with an antibody recognizing activated caspase-3 identifies apoptoticcells in the TIF-IA ${ }^{\text {Nescre }}$ mutants $(\boldsymbol{L})$ but not in controls $(J) \cdot \boldsymbol{G}^{\prime}$ and $\boldsymbol{H}^{\prime}$ represent the magnification of the boxed area. Scale bars: $(\boldsymbol{A}, \boldsymbol{B}) 250 \mu \mathrm{m} ;(\mathbf{C}-\boldsymbol{F}) 500 \mu \mathrm{m} ;(\mathbf{G}-\mathbf{L}) 125 \mu \mathrm{m}$.

47S leader sequence (Qian et al., 2006) (Fig. 2B). Intense prerRNA signals were observed in control hippocampi (Fig. 2C, inset), but not in TIF-IA ${ }^{\text {CaMKCreERT2 }}$ mutants 4 weeks after tamoxifen injection (Fig. $2 D$, inset). At the same stage no major effects on $28 \mathrm{~S}$ rRNA can be detected in TIF-IA CaMKCreERT2 mutants (Fig.
$2 E, F$, insets). Similar results are obtained 2 weeks after tamoxifen injection (data not shown).

When we analyzed $28 \mathrm{~S}$ rRNA 3 months after tamoxifen injection, we observed that decreased rRNA levels occur only in few cells (Fig. 2G,H). By using B23/NPM as marker of nucleolar integrity, we found that in the adult neurons the nucleoli are not equally affected (Fig. $2 I, J)$. In accordance with these findings, increased p53 immunoreactivity was also observed in the mutants, whereas in control mice the p53 levels are below the threshold of immunodetection (supplemental Fig. 1, available at www.jneurosci.org as supplemental material). Interestingly, the cells showing nucleoplasmic distribution of NPM/B23, also show the highest levels of $\mathrm{p} 53$ protein (Fig. $2 \mathrm{~K}, \mathrm{M}, \mathrm{O}$ ), differently from controls (Fig. $2 L, N, P$ ). These experiments indicate that severe nucleolar damage is required for p53 stabilization.

Histological analysis of the hippocampal region by immunostaining with the neuronal marker NeuN did not reveal major differences 2 and 4 weeks after tamoxifen injection between controls and mutants (data not shown). Extensive degeneration in all pyramidal subfields of the hippocampus was detected after 3 months in the mutants (Fig. $3 B, D, F, H$ ) but not in controls (Fig. $3 A, C, E, G$ ). Analysis of the extent of the neurodegenerative damage assessed by counting the number of NeuN positive cells in dentate gyrus, CA1 and CA3 revealed a significant decrease in the mutants 3 months after tamoxifen injection (Fig. 3I).

Neurodegeneration in the hippocampal region of mutant mice was further visualized over time (1,2 and 3months) by FluoroJadeB staining, a fluorescent marker highly specific and sensitive for degenerating neurons (Schmued and Hopkins, 2000) in control and mutant mice (Fig. $4 A, B$ ). We found no differences in the number of FluoroJadeB positive cells 1 month after tamoxifen injection, but positive degenerating cells increased in the mutant 2 and 3 months after injection of tamoxifen (Fig. 4C).

To establish whether the cell loss occurs by apoptosis and determine the rate of cell death over time, we have performed TUNEL assay in control and mutant (Fig. $4 D, E)$. As expected, we found more TUNEL-positive cells 3 months after tamoxifen in the TIF-IA ${ }^{\text {CaMKCreERT2 }}$ mutant mice (Fig. $4 F$ ). However, there was no significant increase in the number of active caspase- 3 positive cells at any of the analyzed time points (data not shown).

Together, these data show that TIF-IA is required for rRNA 


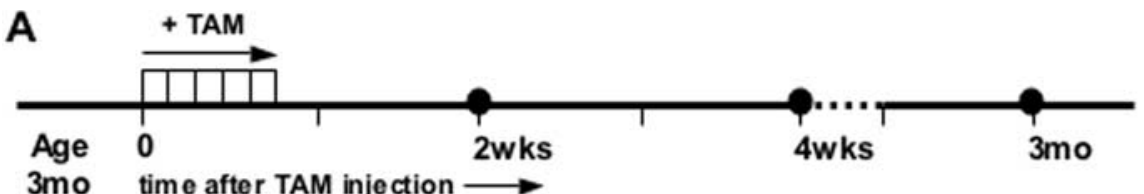

B

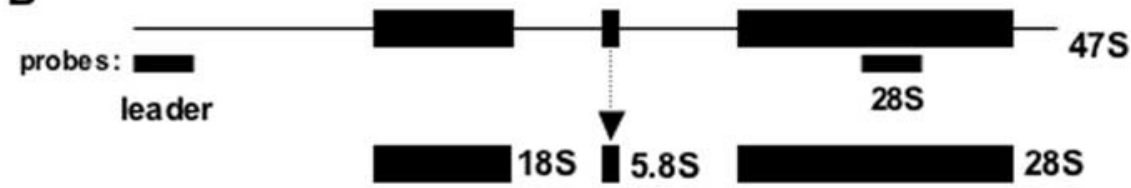

control
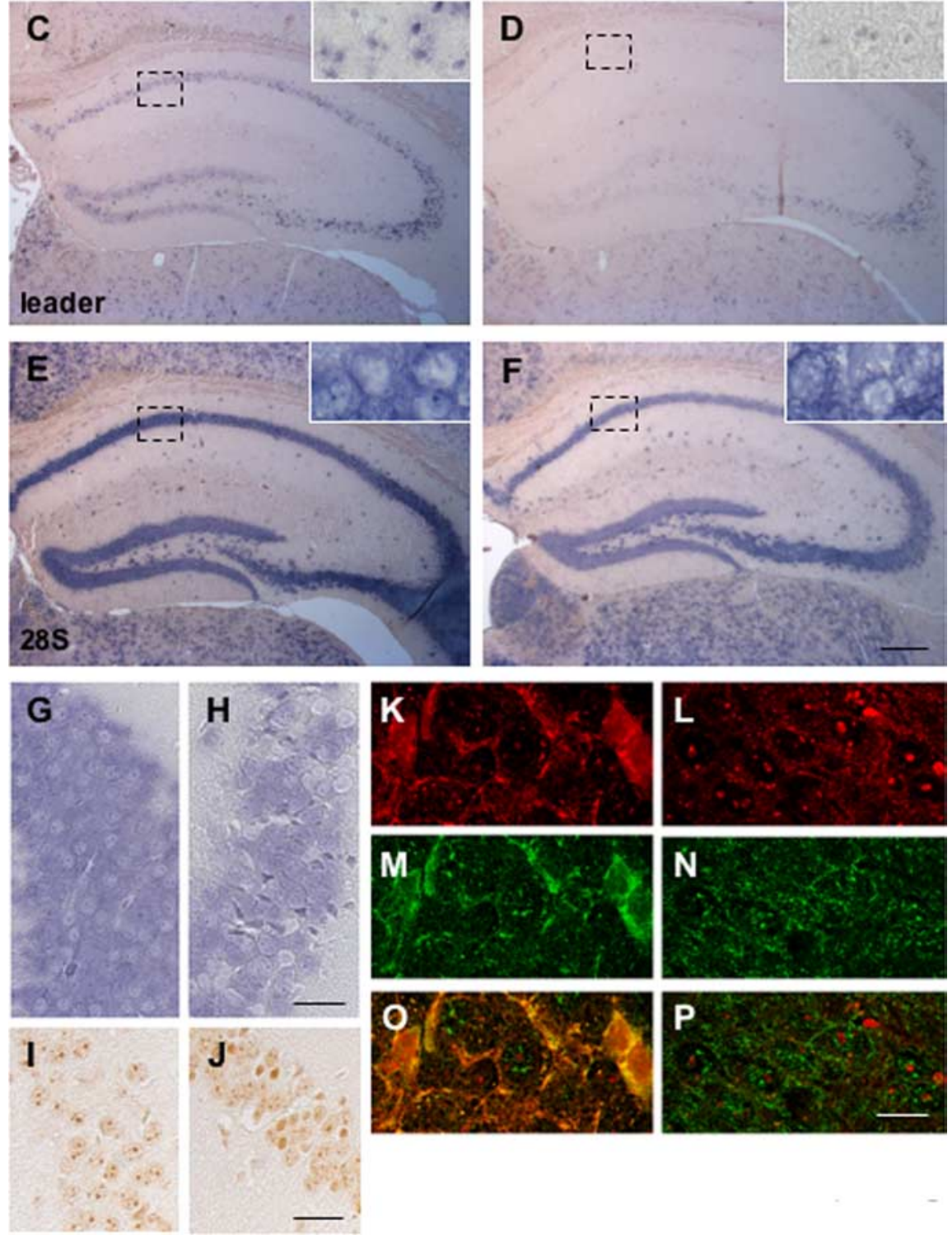

Figure 2. Perturbation of rRNA transcription activity in $T I F-I A^{\text {CaMKCreERT2 }}$ mutants. $A$, Diagram showing the procedure to induce Cre recombinase activity by tamoxifen intraperitoneal injection and the time course of the analysis. $\boldsymbol{B}$, Diagram showing the localization of the probes for the $47 \mathrm{~S}$ leader and the $28 \mathrm{~S}$ rRNA sequence on the rDNA and the final rRNA products. $\mathbf{C}-\boldsymbol{F}$, Nonradioactive in situ hybridization on coronal paraffin sections from control $(\boldsymbol{C}, \boldsymbol{E})$ and $T I F-I A^{\text {CaMKCreERT2 }}$ mutant mice $(\boldsymbol{D}, \boldsymbol{F})$ probed for the 475 pre-rRNA $(\boldsymbol{C}, \boldsymbol{D})$ and $28 \mathrm{~S}$ rRNA $(\boldsymbol{E}, \boldsymbol{F}) 4$ weeks after tamoxifen injection. Insets show high magnification within the boxed field. $\boldsymbol{G}, \boldsymbol{H}$, Nonradioactive in situ hybridization on coronal paraffin sections from control $(\boldsymbol{G})$ and TIF-IA ${ }^{\text {CaMKCreERT2 }}$ mutant mice $(\boldsymbol{H})$ probed for the $28 \mathrm{~S}$ rRNA 3 months after tamoxifen injection. I, J, Immunohistochemistry with NPM/B23 specific antibody transcriptional activity also in differentiated postmitotic neurons, however the consequences of its perturbation on survival are very protracted in comparison with fast dividing cells.

\section{Discussion}

Given its unique ability to regulate RNA polymerase I activity and synthesis of rRNA, we investigated the role of the transcription factor TIF-IA in the developing and adult nervous system. We demonstrate that TIF-IA-dependent functions are essential for the survival of both neural progenitors and nondividing neurons of the adult hippocampus. TIF-IA ablation in proliferating cells leads rapidly to activation of the apoptotic machinery. In contrast, in adult hippocampal neurons, significant signs of neurodegeneration are evident only several months after inducing the mutation, although strong interference with pre-rRNA synthesis occurs already early after induction of the mutation.

Increased levels of the p53 protein are found after TIF-IA ablation in rapidly dividing cells as well as in postmitotic neurons. The precise mechanism by which loss of TIF-IA leads to p53 increase requires further investigation. In neural progenitors one possible mechanism regulating p53 protein levels upon TIF-IA ablation involves the E3 ubiquitin ligase, murine double minute 2 (MDM2), as was already indicated by our study with MEFs (Yuan et al., 2005). Under normal conditions MDM2 regulates p53 turnover by promoting the ubiquitin-proteasome degradation. Alteration of nucleolar integrity and release in the nucleoplasm of nucleolar proteins, such as L11, p19 $9^{\mathrm{ARF}}$ or NPM/ B23 itself, has been shown to interfere with MDM2-p53 interaction resulting in increased stability of p53, thus explaining the relationship between rRNA synthesis, nucleolar integrity and increased p53 levels (Pomerantz et al., 1998; Lohrum et al., 2003; Kurki et al., 2004). Interestingly, loss of MDM2 in neural progenitors by the generation of $M D M 2^{\text {NesCre }}$ mutants leads to phenotypic alterations in the developing brain very similar to those described here in the TIF-IA ${ }^{\mathrm{Nes} C r e}$ mutants (Xiong et al., 2006).

\section{$\leftarrow$}

is used to analyze the nucleoli in control $(I)$ and in the TIF$I A^{\text {NesCre }}$ mutant (J) 3 months after tamoxifen injection. $\boldsymbol{K}-\boldsymbol{P}$, Double immunofluorescence analysis showing NPM/B23 (red) and p53 (green) in TIF-IA CaMKCreERT2 and control 3 months after tamoxifen. Scale bars: (C-F) $500 \mu \mathrm{m}$; (G-J) $125 \mu \mathrm{m}$; (K-P) $50 \mu \mathrm{m}$; (insets) $40 \mu \mathrm{m}$. 

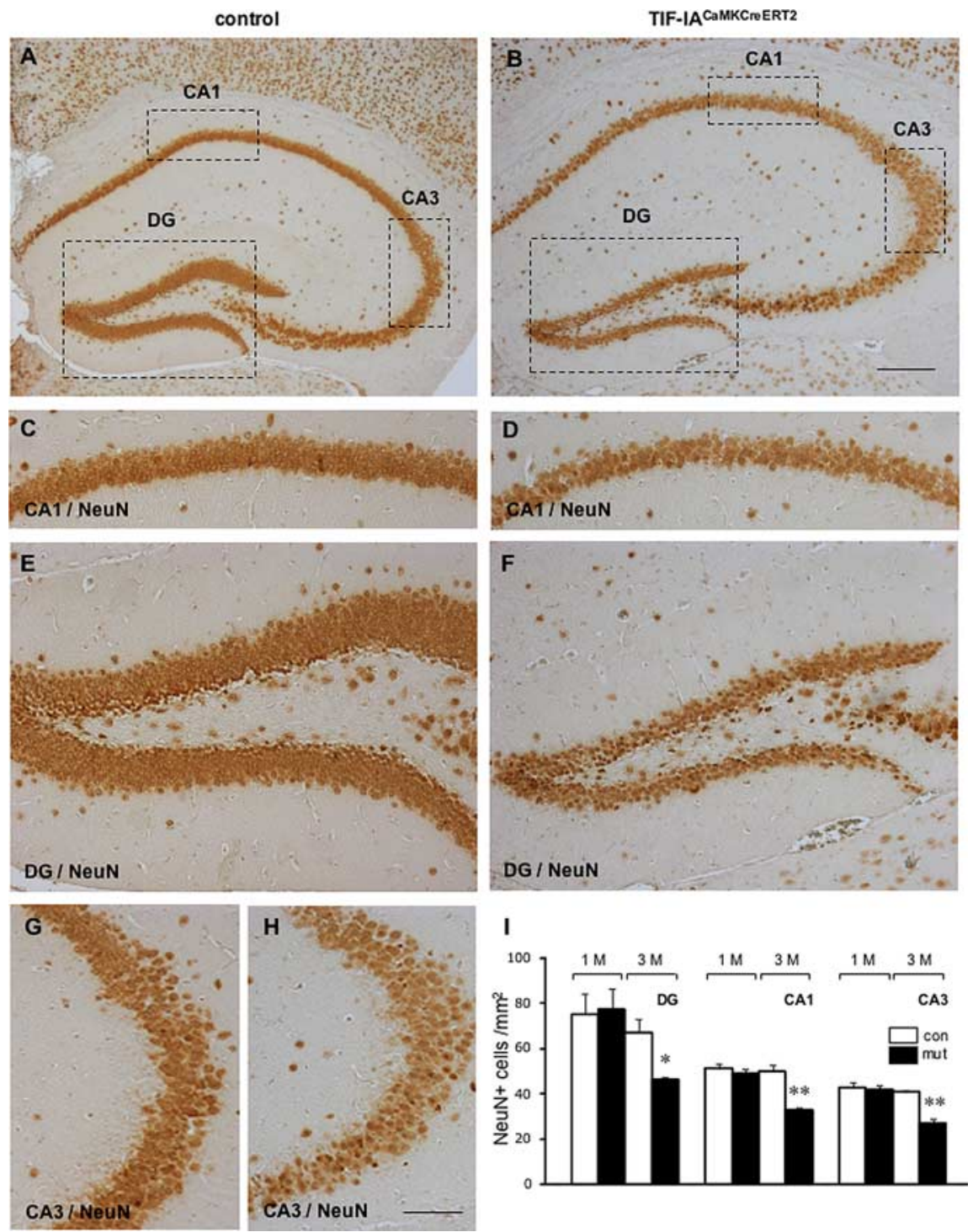

Figure 3. Degeneration of hippocampal neurons in TIF-IA ${ }^{\text {CaMKCreERT2 }}$ mutants. $\boldsymbol{A}, \boldsymbol{B}$, Coronal sections from control and mutant mice were analyzed 3 months after tamoxifen treatment and were immunostained by NeuN specific antibody, a neuronal specific marker. Shown are representative sections from the hippocampal subfields in control $(\boldsymbol{C}, \boldsymbol{E}, \boldsymbol{G})$ and mutant mice $(\boldsymbol{D}, \boldsymbol{F}, \boldsymbol{H})$. I, Quantification of NeuN-positive cells in the dentate gyrus (DC), CA1 and CA 3 hippocampal subfields of control and TIF-IA ${ }^{\text {CaMKCreERT2 }}$ mutants 1 and 3 months after tamoxifen injection ( $n=3-4$ mice per genotype). Error bar represents \pm SEM. Statistical significance was analyzed using a homoscedastic Student's $t$ test. Values were considered significantly different with ${ }^{*} p<0.05$ and ${ }^{* *} p<0.01$. Scale bars: $(\boldsymbol{A}, \boldsymbol{B}) 500 \mu \mathrm{m} ;(\boldsymbol{C}-\boldsymbol{H}) 125 \mu \mathrm{m}$.

The observation that adult hippocampal neurons are responding more slowly to the effects of rRNA synthesis perturbation raises intriguing questions about the specific requirement of nucleolar functions in different cellular contexts. A recent study shows that prolonged neuronal activity influences the generation of functional nucleoli in postmitotic cultured hippocampal neurons, thereby regulating protein synthesis and identifies the protein AIDA-1d as a regulator of nucleolar assembly (Jordan et al., 2007). Nevertheless, the functional complexity of the nucleolus is strongly underscored by the identification of a number of proteins that shuttle between the nucleolus and the nucleoplasm with functions beyond ribosome biogenesis (Andersen et al., 2005; Martindill et al., 2007).

Our study indicates that the genetic ablation of TIF-IA leads to chronic neurodegeneration in mice. As of yet, efficient and rapid cell ablation has been achieved in vivo by the use of diphtheria toxin that inactivates the elongation factor 2 resulting in termination of protein synthesis and apoptosis of the targeted cells (Brockschnieder et al., 2004). By ablation of TIF-IA we do not interfere directly with protein synthesis, but with synthesis of rRNA and nucleolar integrity. In this way, we influence a pathway which is of major significance in the control of cellular survival. The activity of Pol I may be of significance for a certain cell type as reflected by the Klotho insufficiency, in which anterior horn cell degeneration occurs as a consequence of decreased rRNA synthesis (Anamizu et al., 2005) or as reflected by the Tcof1 haploinsufficiency which leads to perturbation of ribosome biogenesis and is associated with a congenital disorder of craniofacial development (Jones et al., 2008).

Our results gives the possibility to use targeted deletion of the TIF-IA gene as a tool to trigger cell death by an endogenous suicide mechanism. Thus, to get insights into the effects of nucleolar perturbation on neurodegenerative processes, it will be useful to generate mutants lacking TIF-IA in neurons degenerating in severe diseases, e.g., dopaminergic neurons in Parkinson's disease.

\section{References}

Anamizu Y, Kawaguchi H, Seichi A, Yamaguchi S, Kawakami E, Kanda N, Matsubara S, Kuro-o M, Nabeshima Y, Nakamura K, Oyanagi K (2005) Klotho insufficiency causes decrease of ribosomal RNA gene transcription activity, cytoplasmic RNA and rough ER in the spinal anterior horn cells. Acta Neuropathol 109:457-466.

Andersen JS, Lam YW, Leung AK, Ong SE, Lyon CE, Lamond AI, Mann M (2005) Nucleolar proteome dynamics. Nature 433:77-83.

Borer RA, Lehner CF, Eppenberger HM, Nigg EA (1989) Major nucleolar proteins shuttle between nucleus and cytoplasm. Cell 56:379-390.

Brockschnieder D, Lappe-Siefke C, Goebbels S, Boesl MR, Nave KA, Riethmacher D (2004) Cell depletion due to diphtheria toxin fragment A after Cre-mediated recombination. Mol Cell Biol 24:7636-7642.

Erdmann G, Schütz G, Berger S (2007) Inducible gene inactivation in neurons of the adult mouse forebrain. BMC Neurosci 8:63.

Grummt I (2003) Life on a planet of its own: regulation of RNA polymerase I transcription in the nucleolus. Genes Dev 17:1691-1702.

Jones NC, Lynn ML, Gaudenz K, Sakai D, Aoto K, Rey JP, Glynn EF, Ellington L, Du C, Dixon J, Dixon MJ, Trainor PA (2008) Prevention of the neurocristopathy Treacher Collins syndrome through inhibition of p53 function. Nat Med 14:125-133.

Jordan BA, Fernholz BD, Khatri L, Ziff EB (2007) Activity-dependent AIDA-1 nuclear signaling regulates nucleolar numbers and protein synthesis in neurons. Nat Neurosci 10:427-435.

Kalita K, Makonchuk D, Gomes C, Zheng JJ, Hetman M (2008) Inhibition of nucleolar transcription as a trigger for neuronal apoptosis. J Neurochem. Advance online publication. Retrieved November 7, 2008. doi: 10.1111/j.1471-4159.2008.05316.x

Kurki S, Peltonen K, Latonen L, Kiviharju TM, Ojala PM, Meek D, Laiho M (2004) Nucleolar protein NPM interacts with HDM2 and protects tumor 

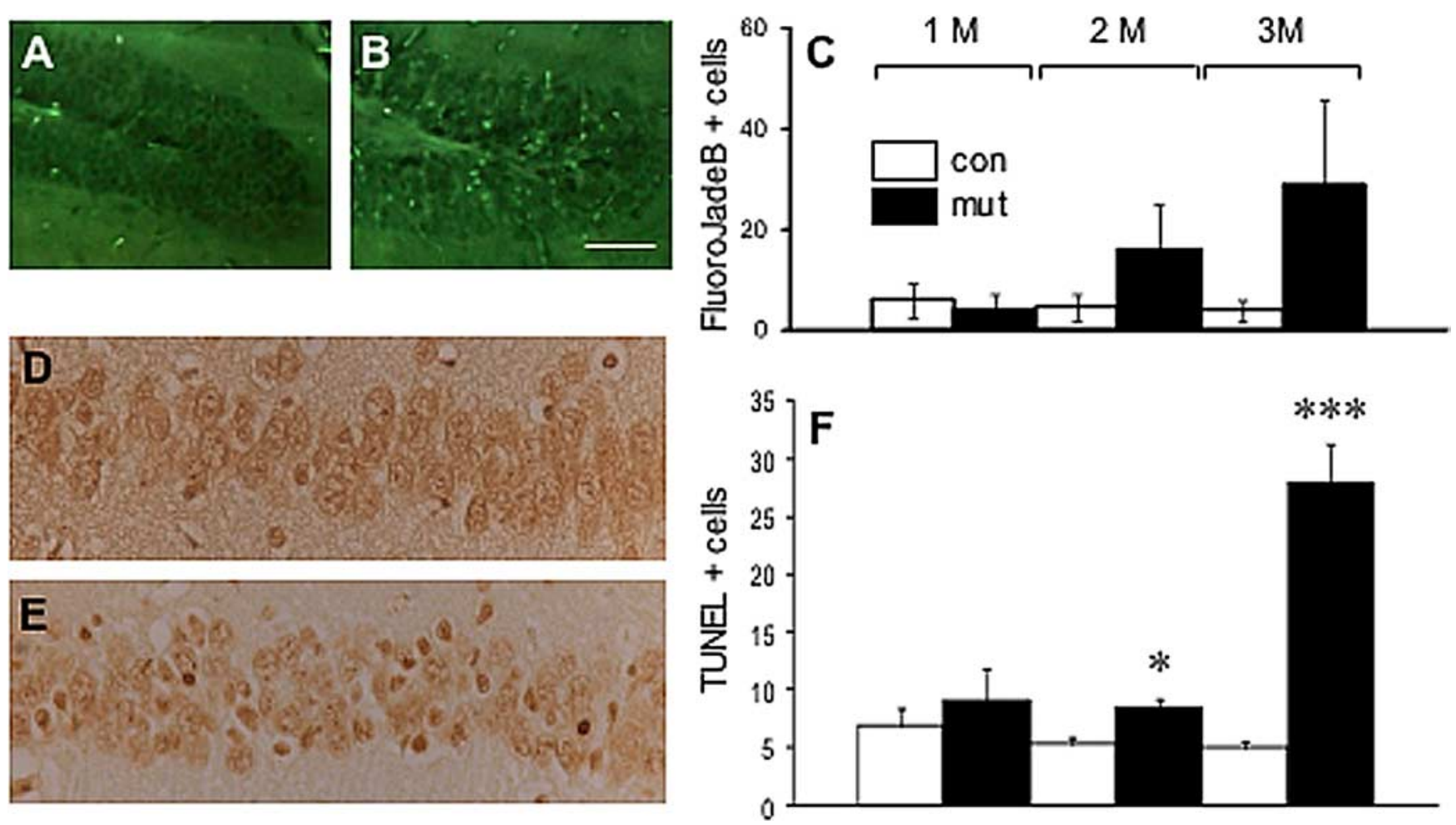

Figure 4. $\quad A, B$, Fluoro-Jade B staining identifies dead or dying neurons in the DG of control and TIF-IA ${ }^{\text {CaMKKreERT2 }}$ mutant. C, Quantification of FluoroJade-positive cells in the hippocampus 1,2 and 3 months after tamoxifen injection. D, E, TUNEL-positive cells in the CA1 of control and TIF-IA ${ }^{\text {CaMKCreERT2 }}$ mutants. F, Quantification of TUNEL-positive cells 1,2 and 3 months after tamoxifen injection ( $n=3$ mice per genotype). ${ }^{*} p<0.05 ;{ }^{* * *} p<0.001$. Scale bars: $(\boldsymbol{A}, \boldsymbol{B}) 150 \mu \mathrm{m} ;(\boldsymbol{C}, \boldsymbol{D}) 100 \mu \mathrm{m}$.

suppressor protein p53 from HDM2-mediated degradation. Cancer Cell 5:465-475.

Lohrum MA, Ludwig RL, Kubbutat MH, Hanlon M, Vousden KH (2003) Regulation of HDM2 activity by the ribosomal protein L11. Cancer Cell 3:577-587.

Martindill DM, Risebro CA, Smart N, Franco-Viseras Mdel M, Rosario CO, Swallow CJ, Dennis JW, Riley PR (2007) Nucleolar release of Hand 1 acts as a molecular switch to determine cell fate. Nat Cell Biol 9:1131-1141.

Mayer C, Bierhoff H, Grummt I (2005) The nucleolus as a stress sensor: JNK2 inactivates the transcription factor TIF-IA and down-regulates rRNA synthesis. Genes Dev 19:933-941.

Olson MO (2004) Sensing cellular stress: another new function for the nucleolus? Sci STKE 2004:pe10.

Parlato R, Rosica A, Rodriguez-Mallon A, Affuso A, Postiglione MP, Arra C, Mansouri A, Kimura S, Di Lauro R, De Felice M (2004) An integrated regulatory network controlling survival and migration in thyroid organogenesis. Dev Biol 276:464-475.

Pomerantz J, Schreiber-Agus N, Liégeois NJ, Silverman A, Alland L, Chin L, Potes J, Chen K, Orlow I, Lee HW, Cordon-Cardo C, DePinho RA (1998)
The Ink4a tumor suppressor gene product, p19Arf, interacts with MDM2 and neutralizes MDM2's inhibition of p53. Cell 92:713-723.

Qian J, Lavker RM, Tseng H (2006) Mapping ribosomal RNA transcription activity in the mouse eye. Dev Dyn 235:1984-1993.

Rubbi CP, Milner J (2003) Disruption of the nucleolus mediates stabilization of $\mathrm{p} 53$ in response to DNA damage and other stresses. EMBO J 22:6068-6077.

Schmued LC, Hopkins KJ (2000) Fluoro-Jade B: a high affinity fluorescent marker for the localization of neuronal degeneration. Brain Res 874:123-130.

Tronche F, Kellendonk C, Kretz O, Gass P, Anlag K, Orban PC, Bock R, Klein R, Schütz G (1999) Disruption of the glucocorticoid receptor gene in the nervous system results in reduced anxiety. Nat Genet 23:99-103.

Xiong S, Van Pelt CS, Elizondo-Fraire AC, Liu G, Lozano G (2006) Synergistic roles of $\mathrm{Mdm} 2$ and $\mathrm{Mdm} 4$ for $\mathrm{p} 53$ inhibition in central nervous system development. Proc Natl Acad Sci U S A 103:3226-3231.

Yuan X, Zhou Y, Casanova E, Chai M, Kiss E, Gröne HJ, Schütz G, Grummt I (2005) Genetic inactivation of the transcription factor TIF-IA leads to nucleolar disruption, cell cycle arrest, and p53-mediated apoptosis. Mol Cell 19:77-87. 